\title{
ReactionCode: a new versatile format for searching, analysis, classification, transform, and encoding/decoding of reactions
}

\author{
Victorien Delannée ${ }^{1}$ and Marc C. Nicklaus ${ }^{1}$ \\ ${ }^{1}$ Computer-Aided Drug Design Group, Chemical Biology Laboratory, Center for \\ Cancer Research, National Cancer Institute, NIH, Frederick, MD 21702, USA
}

March 2020

\section{Abstract}

In the past two decades a lot of different formats for molecules and reactions have been created. These formats were mostly developed for the purposes of identifiers, representation, classification, analysis and data exchange. A lot of efforts have been made on molecule formats but only few for reactions where the endeavors have been made mostly by companies leading to proprietary formats. Here, we developed a new open-source format which allows to encode and decode a reaction into multi-layers machine readable code, which aggregates reactants and products into a condensed graph of reaction (CGR). This format is flexible and can be used in a context of reaction similarity searching and classification. It is also designed for database organization, machine learning applications and as a new transform reaction language.

\section{Introduction}

Different proprietary and open formats for reactions have been invented over the past 50 years. The first reaction format can probably be attributed to E.J. Correy and W.T. Wipke. They implemented a format based on rules to generate new molecules and integrated it in the first computer-aided organic synthesis program: OCSS (Organic Chemical Simulation of Synthesis). [3] This project split to give birth to LHASA (Logic and Heuristics Applied to Synthetic Analysis) $[2,4][18]$ and SECS (Simulation and Evaluation of Chemical Synthesis) [23]. The LHASA team designed the language CHMTRN (CHeMistryTRaNslator), while the SECS group created the ALCHEM (A Language for CHEMistry) language. [24] After their launch, diverse additional reaction transform languages came up along the implementation of programs such as CLASS and IGOR 
\& IGOR2. However, the arrival of SMILES (Simplified Molecular Input Line Entry System) in the late 1980s led to the development of ReactionSMILES and SMIRKS (SMIles ReaKtion Specification). These two formats were largely adopted by the community and are still widely used nowadays. [14, 20, 21, 1]

The work around reaction formats has also affected the need for representations and identifiers for data exchange. In the 1990s, Molecular Design Limited (MDL) developed the Chemical Table file (CTfile) format. [5] In this context, the RXNfile and RDfile formats were defined with the objective to store reaction data and quickly became a reference. RXNfile is used to store the structural information for the reactants and products of a single reaction [5], while RDFile allows one to store a set of RXNs with their associated data. [5] Since then, additional formats have emerged or are under development such as XDfile, MRV, UDM, CMLReact, CDX/CDXML and ReactionSPL. However, none of these formats succeeded in establishing itself widely as the CTfile formats are still much more frequently used. Next to these representations, work on reaction identifiers was also done. The Reaction International Chemical Identifier (RInChI) [10], an application of InChI [11, 12] was recently developed with the objective to offer a unique reaction identifier, which can help to organize and validate reaction databases. [10]

Besides the formats specifically designed to describe reaction transforms and allow easy data exchange, other more versatile formats have been developed in order to try to offer more flexibility and be utilized in different contexts related to reactions. In 1986, Fujita proposed the Imaginary Transition State (ITS) format, which aggregates reactants and products inside a pseudo-molecule in which the bond changes of a reaction are annotated. This pseudo-molecule was created to be used for the purposes of reaction retrieval and design. [8] This format evolved and became known as Condensed Graph of Reaction (CGR). Stored in an SD File, it is mainly employed for machine learning applications, similarity search, and classification. [13,6] Unfortunately, this format cannot be employed as-is and all analysis methods using it are based on molecular graph coloration and molecular fragment generated from the CGR [19, 6, 17, 9]. Next to the CGR format, three multi-layer formats considering the reaction center and the neighbor atoms have been developed by J.L. Faulon, InfoChem and Elsevier. J.L. Faulon created the reaction signature, where each reactant and product are described as a tree without taking into account the bond type, and calculates the difference between the reactant and products trees. [7] Despite the versatility of this approach, the consideration of only the atom types and their simple connection is a huge limitation. InfoChem developed the reaction ClassCode, which provides a unique identifier (hash) for the reaction center and its two closest atom neighborhood layers. [16] Similarly, Elsevier implemented the BINCODE, which computes, using a pseudo-molecule, a linear string for each layer from the reaction center to the deepest atom neighborhood layers. Each layer contains the atoms that compose it and their connection tables. In addition, the BINCODE also encodes the bond fate and the atom hybridization 
change. While the ClassCode is limited to a depth of 2 and is strict by its nature as an identifier, the BINCODE appears to offer more flexibility. Indeed, it covers the complete reaction, and its nature as a string allows some modifications for search purposes. However, the BINCODE was made to be overly generalist by encoding elements into categories (e.g. the halogens $\mathrm{Cl}, \mathrm{Br}$, and I have the same encoding). It therefore cannot be used to recover the entire reaction.

To overcome these limitations, we have developed a new format named ReactionCode, which is a multi-layer machine readable code. This open source format is canonical and designed to be flexible, upgradeable and versatile in order to be applied in a broad range of applications. ReactionCode is particularly useful for reaction similarity searching and classification, but is also conceived for machine learning applications and as a new transform reaction language.

\section{ReactionCode format}

\subsection{Structure}

The ReactionCode is a multi-layer machine readable code, which is produced from the aggregation of reactants and products into a condensed graph of reaction (CGR) (Figure 1). The ReactionCode is organized into three blocks, which contain their corresponding layers:

\section{Reaction center}

2. Atoms around the reaction center remaining in the product

3. Leaving atoms around the reaction center (if any)

Each layer is composed of a main sub-layer and up to three optional sub-layers, which describes the stereochemistry, the charges, and the isotope, respectively. A layer starts with a number if it illustrates the reaction center or the remaining group, or a letter if it describes the leaving group. It is always terminated by the symbol ' ''.

\subsubsection{Main sub-layer}

The main sub-layer is composed of 4 types of information: the depth, the atom code, the connection table and the atom stoichiometry. This layer starts with the depth followed by ' $:$ '. The depth indicates the distance relative to the reaction center. It is expressed in numbers for the reaction center and the remaining group(s) and in letters for the leaving group(s). The atom code is composed of three characters: the first indicates the highest status of the connected bonds encoded using the hexadecimal system (Table S7), the two others encode the atom type (Table S8). Each atom code is followed by a parenthesized connection table, which indicates each bond connected to an atom with a lower index. A bond is encoded by 4 characters: the 1st indicates the bond order in reactants, 


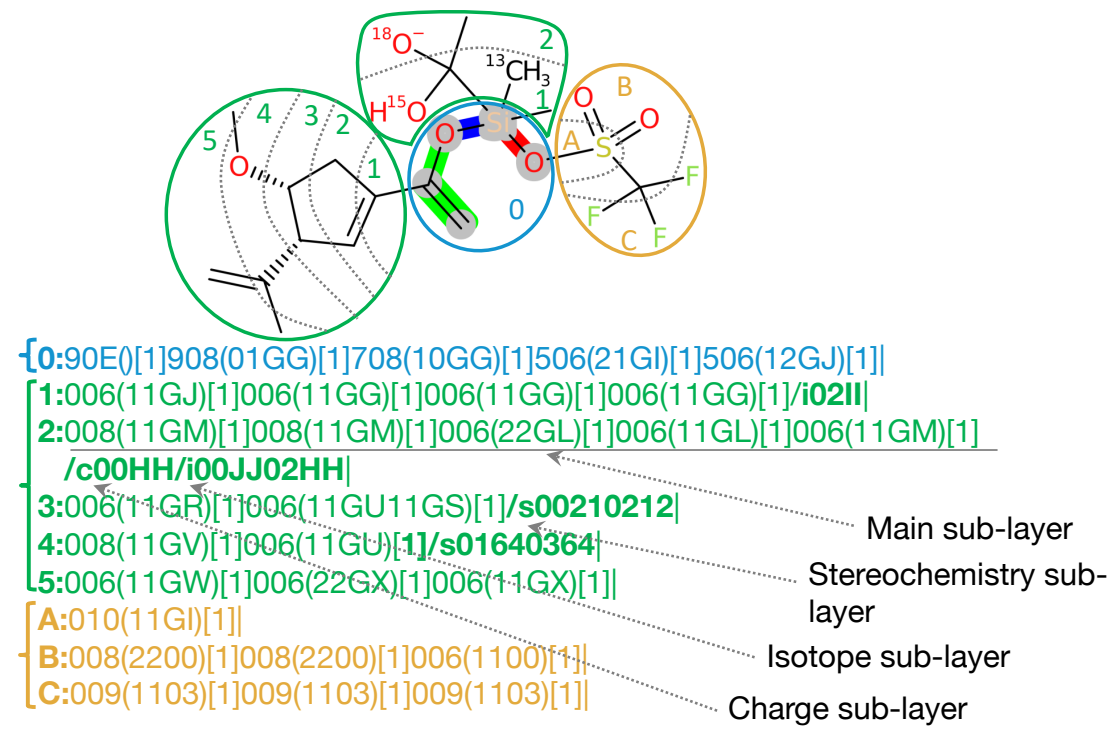

\section{Blocks:}

The reaction centre

The atoms around the reaction centre remaining in products

The atoms around the reaction centre leaving (if present)

Figure 1: ReactionCode structure

The ReactionCode is composed of three blocks. The first one describes the reaction center (shown in blue) and starts with '0:', which corresponds to depth 0 . The entire reaction center (atoms highlighted in gray) is always stored in this single layer. The block in green illustrates the atoms around the reaction center that are still kept in the product(s). This block can be composed of one or multiple layers and each layer starts with a number followed by ':'. The figure indicates the depth of the atoms present in this layer in relation to the reaction center. The block shown in yellow encapsulates the atoms around the reaction center which are absent in the product(s). This block can be composed of one or multiple layers and each layer starts with a letter followed by ':'. The figure indicates the depth of the atoms present in this layer in relation to the reaction center. (The letter A means a depth equal to one.)

Each layer is terminated by a 'l' symbol and is composed of a main sub-layer that starts after the : symbol. The optional layers begin with the '/' symbol. /s characterizes the stereochemistry layer. /c describes the charge layers. /i indicates the isotope layer. 
the $2 \mathrm{~d}$ encodes the bond order in products (Table S6) and the last two refer to the index of the other atom connected to. The indices are encoded using the hexadecimal system for the atoms to connect that are present in the blocks corresponding to the leaving group (Table S1) and the indices of atoms in the two other blocks are encoded using a lookup table (Table S2). Finally, the square brackets store the atom stoichiometry, i.e. the number of times the same atom is in the products (Example in SI Figure S1).

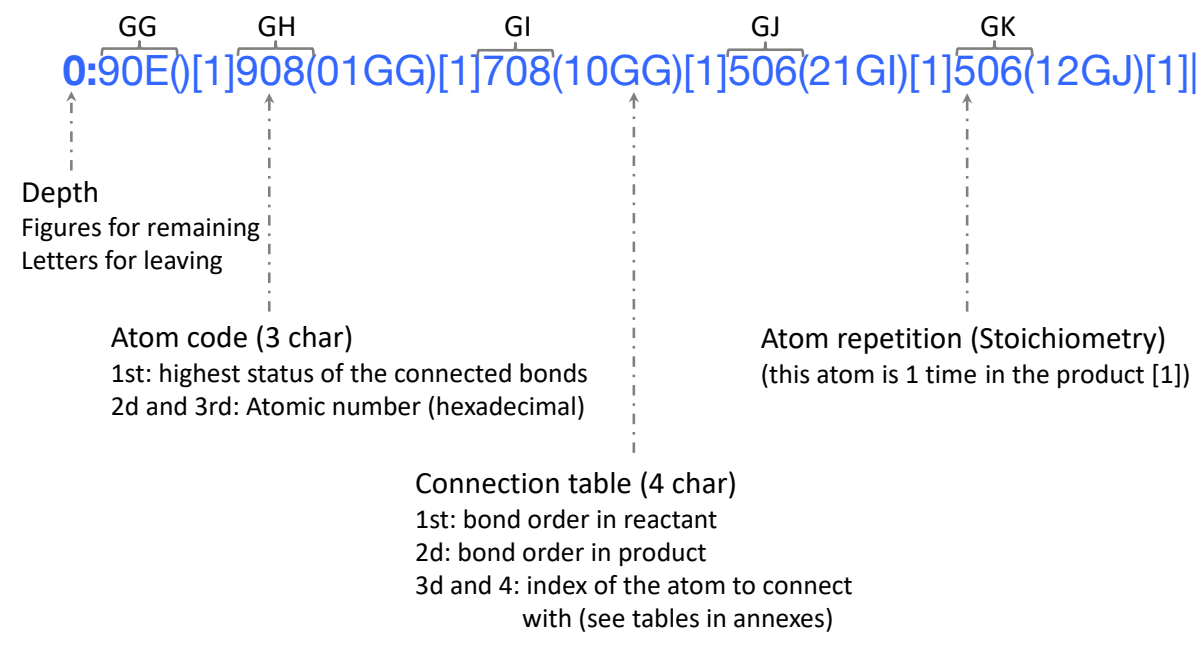

Figure 2: Main sub-layer composition

A layer starts with its depth, which is the distance of its atoms from the reaction center (which has depth $=0$ ). The next three characters characterize the atom. The first character indicates the highest bond status among all bonds connected to this atom. (Table S7. For instance, the $\mathrm{Si}$ atom (90E) is connected by 4 single bonds: 2 are not part of the reaction centre and are encoded by $0 ; 1$ bond is broken, encoded by 7 ; and another one is made, encoded by 9 , which is the highest bond status. The two other characters encode the atom symbol (Table S8). E.g., 0E stands for Si. The connection table is contained between the brackets. Each bond in the connection table is encoded by 4 characters. E.g., the connection table (11GU11GS) encodes 2 bonds. The first two characters indicate the bond order in reactants for the first one and in products for the second one, respectively (Table S6). 01GG means a bond is made with the atom at index GG. The last two characters represent the index of the other atom to connect to (see Table S2 for the reaction center and the remaining group and Table S1 for the leaving group). The square brackets store the atom stoichiometry, i.e. the number of times a same atom is in the products (Example in SI Figure S1). 


\subsubsection{Optional sub-layers}

The optional sub-layers qualify the atom and bond in their corresponding layer. Only the sub-layer(s) where a change has to be made are written directly after the end of the main sub-layer. The priority order is: 1) the charge sub-layer $(/ \mathrm{c})$, 2 ) the stereochemistry sub-layer (/s) and 3 ) the isotope sub-layer (/i) (Figure 3 ).

Stereochemistry layer The stereochemistry layer starts with /s and the relative information is contained in a block containing the atom or bond index (2 digits), which has the corresponding stereochemistry modification and 2 characters encoding the stereochemistry in reactants by the first character and in products by the second one (Tables S3 and S4). E.g., in /s01640364," /s" indicates that this layer contains stereochemistry information. It having 8 characters means that $2(8 / 4)$ entities (atom(s) and/or bond(s)) have a stereochemistry information. The two modifications are: "0164" and "0346". The first modification is encoded by the first 4 characters "0164". 01 means that the entity at index 01 , which is the bond "11GV", is modified. The third character "4" encodes a DOWN bond in reactants, which becomes an UP bond in products indicated by the fourth character " 6 ". The next 4 characters 0364 modify the bond "11GU" from UP to DOWN.

Charge layer The charge layer starts with /c and the charge information is contained in a block containing the charged atom index ( 2 digits) and 2 characters encoding the charge. The first one encodes the state in reactants and the second one the change in products (Table S5). E.g., in /c00HH, "/c" indicates that this layer contains charge information. It having 4 characters means that 1 (4/4) atom has a charge. The only modification is: "00HH". 00 means that the entity at index 00 , which is the atom "008", is modified. The third character " $H$ " encodes a negative charge -1 , which remains unchanged in products as the fourth character is encoded by the same letter" $\mathrm{H}$ ".

Isotope layer The isotope layer starts with / $\mathrm{i}$ and the isotope information is contained in a block containing the isotope atom index (2 digits) and 2 characters encoding the mass difference between the current isotope and the reference. The first one is for the reactants and the second one for products (Table S5). E.g., in /i00JJ02HH "/i" indicates that this layer contains isotope information. It having 8 characters means that $2(8 / 4)$ atoms are isotopes. The two modifications are: "00JJ" and "02HH". 00 means that the entity at index 00 , which is the atom " 008 ", is modified. The third character " J" encodes an addition of 2 neutrons to the common isotope. 008 encodes an oxygen with 2 more neutrons, which means that the atom is an ${ }^{18} \mathrm{O}$. The fourth character " $\mathrm{H}$ " is unchanged, which indicates that the atoms in products remains the same isotope. 

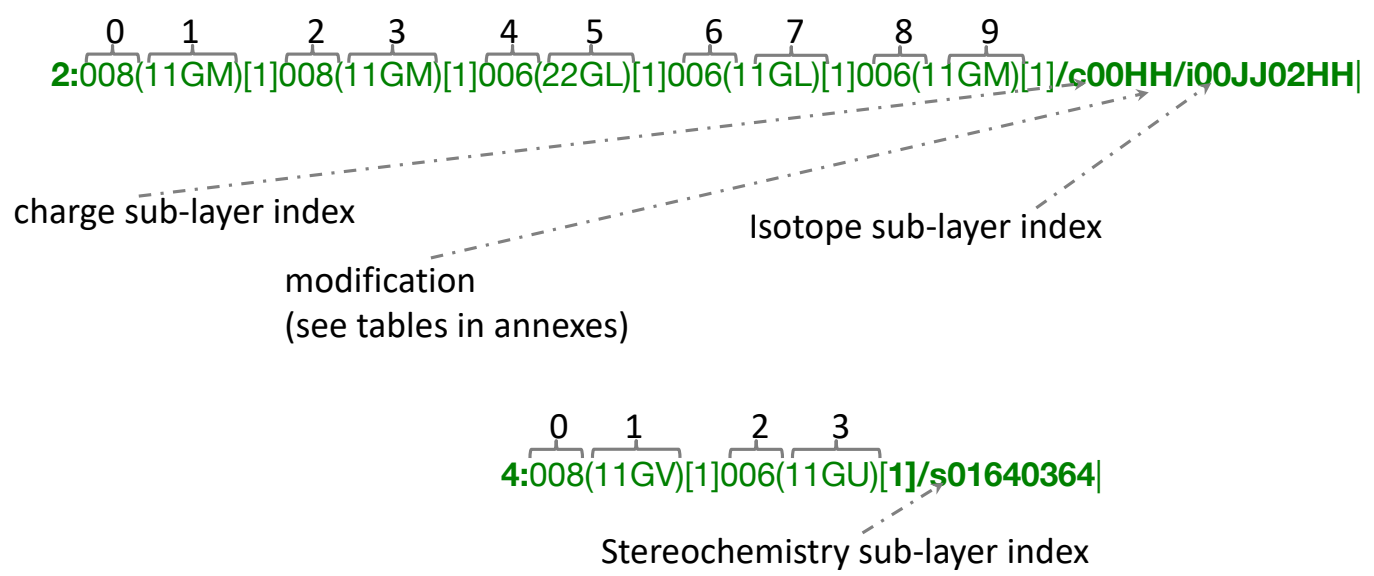

Figure 3: Optional sub-layer composition

An optional sub-layer is directly located after the main sub-layer. Such a code is composed by the symbol '/' followed by a letter and indicates the modification (/c for charge, /i for isotope and /s for stereochemistry). Each block qualifying an atom or a bond is composed of four characters. The first two characters are a number ( 2 characters) indicating the index (decimal system) of the entity (atom or bond) in the current layer which has to be modified. The next 2 characters are encoding the change to apply to reactants (first character) and products (second character) (for isotope and charge see table S5; for atom stereochemistry see table S3; and for bond stereochemistry, see table S4). 


\subsection{Encoding/Decoding process}

One of the major strengths of ReactionCode is its capacity to be bidirectional: a reaction encoded into ReactionCode can be easily partially or fully decoded to get the reaction back (Figure 4).

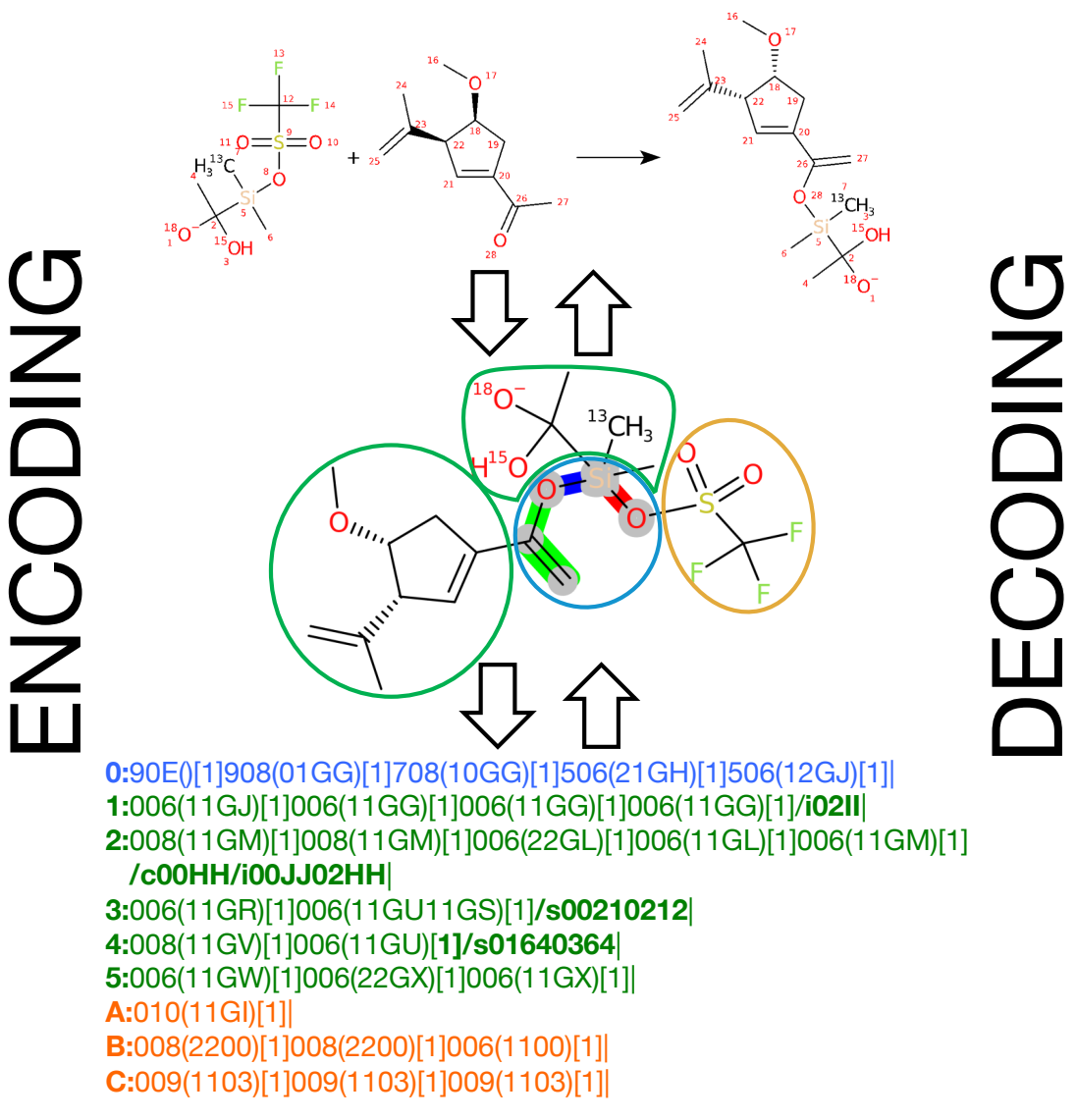

Figure 4: Encoding and decoding process

Encoding: The reactants and products of the mapped reaction are aggregated into a pseudo-molecule. The bond changes are annotated: green for a bond order change, blue for a bond made, and red for a bond broken. All atoms and bonds are annotated as part of the reaction center (inside blue circle), remaining in the final product (green circles), or leaving the final product (yellow circle). Finally, all atoms and bonds are encoded into ReactionCode by layers starting from the reaction center to the outermost layer.

Decoding: The ReactionCode is transformed into a pseudo-molecule, which allows one to recover the initial reaction. 
In order to generate the ReactionCode, a mapped reaction is necessary. The first step consists in annotating each atom and bond in reactants and products. Three types of annotation are computed:

- atoms and bonds constituting the reaction center

- atoms and bonds present both in reactants and products, which are annotated as the remaining group

- atoms and bonds present in reactants but absent in products (if any), which are annotated as the leaving group

Once the annotation part is finished, reactants and products are aggregated into a CGR. Finally, the ReactionCode is generated from the CGR. Each atom of the CGR is encoded and reverse-ranked by layers. The algorithm starts from the reaction center, reverse-ranks each atom of this layer and makes the connection between them. Then, a Breadth First Search (BFS) algorithm is used to obtain all the surrounding atoms having a depth of 1 . These atoms are separated into 2 layers: those belonging to the remaining layer and those that are part of the leaving group. All encoded atoms are reverse-ranked and the connections between each atom with the current and the previous layer are established. The algorithm iterates this procedure until all atoms have been visited (Figure 5).

The decoding process reconstructs the pseudo-molecule from the ReactionCode by transforming each atom code into an atom object and making the bonds between them. This step relies on the chemoinformatics Java libraries contained in CDK (Chemistry Development Kit)[22]. Then, the pseudo-molecule is transformed into reactants and products in order to get the original reaction back. The ReactionCode is set up by default to recover a balanced reaction but the elements present in the leaving group block could be ignored by the user in order to not have them in the products.

\section{ReactionCode software}

Java powered by CDK was used to develop the software to generate the ReactionCode, to decode it, to make pseudo-molecules, and to use it as a new transform language. All these functions can be easily used thanks to a CLI (command line interface) and the JAR file can also be directly employed as an API by calling the corresponding class. In addition, this tool has been successfully tested on open source reaction dataset from the USPTO (https://bitbucket.org/dan2097/patent-reaction-extraction/downloads), for which all reactions were successfully encoded.

Encoder The encoder allows one to produce the pseudo-molecules and ReactionCodes. It takes the most common formats as input: SMIRKS (single or a set of SMIRKS in a file), RXN and RDF. The encoder can provide the pseudosmiles in SDF and in SMILES format and depict them. Finally, the generated 


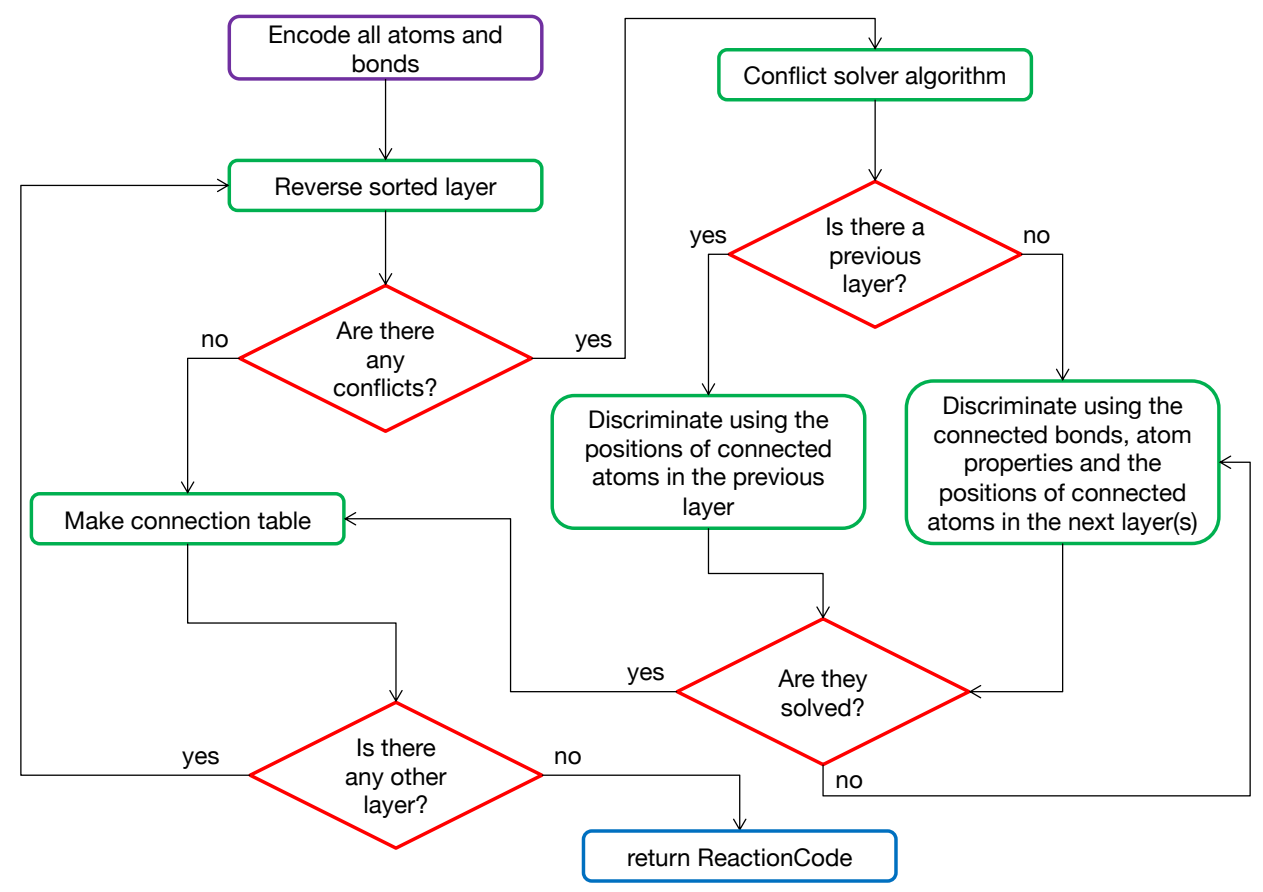

Figure 5: ReactionCode encoding algorithm

Once all atoms and bonds are encoded, all encoded atoms present in the reaction center $($ depth $=0)$ are reverse sorted. If two or more atoms have the same code, the conflict solver algorithm is started. The conflict algorithm will make a tree of the connected atom (see Figure S2 in Supplementary Information) and compare the atom codes and the bond codes of the atom present in the next layer using a BFS algorithm. The algorithm iterates until the conflicts are solved and a proper order can be set up. Then, the connections between the atoms in the current layer are made. Then, all the atoms present in the depth $\mathrm{n}+1$ (if any), which will be all atoms connected with those in the reaction center in this situation, are reverse sorted. If there are any conflicts, they are first solved by comparing the position of the connected atoms in the previous layer, then using the connected bonds, the atom properties (stereochemistry, charge and isotopy) and finally in the next layer(s) if the conflict cannot be solved. We iterate this process until all layers are processed. 
ReactionCodes are given in a CSV file.

Decoder The decoder allows one to get the original reaction back. The reactions can be provided as reactionSMILES, SMIRKS, RXN, or RDF. They can also depicted as a PNG file. In addition, a partial reaction can be generated by giving the layers of interest as input (Figure 6).

Transformer Thanks to the structure of ReactionCode, where each layer is only dependent on its previous layers but independent from its subsequent layers, it can be used as a transform language where the ReactionCode is transformed into a pattern applied to a set of reactants (Figure 6). The transformer takes a complete or partial ReactionCode (set of layers) and the reactants as a unique SMILES String or an SD file. The transformer will generate all unique possible products and outputs them in reactionSMILES, SMIRKS, RXN, or RDF format.

\section{$5 \quad$ ReactionCode applications}

ReactionCode is a format that can be used for multiple purposes. We describe a few of them here.

\subsection{Searching for similar reactions}

The ReactionCode is perfectly suited to search for similar reactions in a database as it is in string format. In addition, a wild card can replace each figure or letter. This can be employed in order to match with any atom, or to ignore the bond order, or any property desired by the user. The syntax of the ReactionCode thus provides the user with a broad flexibility.

\subsection{Reaction transform language}

The ReactionCode is also designed as a new reaction transform language. One or multiple layers can be used to match a set of reactants in order to generate all the possible products and get all possible reactions. This can be easily done by using our software. Note, however, that this approach does not incorporate any knowledge about the actual synthetic accessibility of the proposed reaction (in contrast to CHMTRN/PATRAN [15]) but operates strictly on the basis of pattern matching.

\subsection{Classification}

The structure by layers of the ReactionCode allows one to classify the reaction in order to make statistical analyses, study the diversity or just to have an idea 
Layer 0

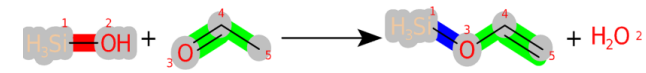

Layers $0+1$

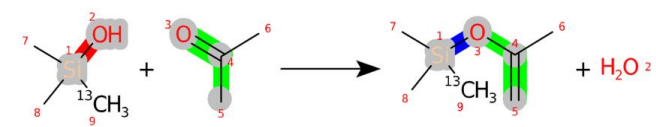

Layers $0+1+\mathrm{A}$

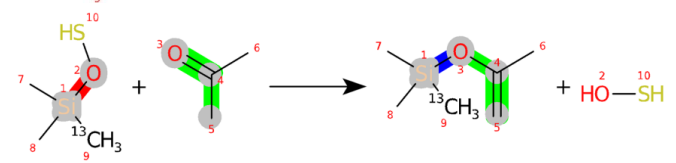

Layers $0+1+2$

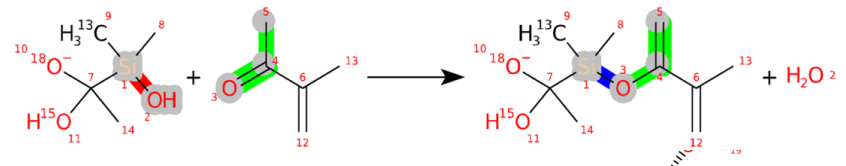

Layers $0+1+2$

$+A+B$

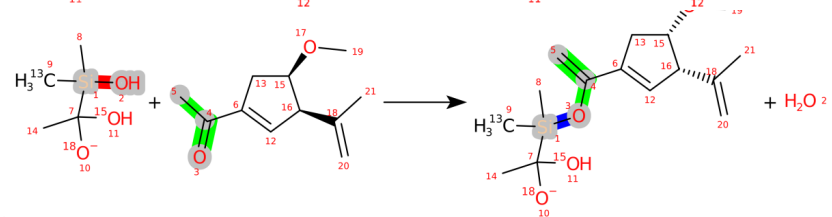

Layers $0+1+2+3$

$+4+5$

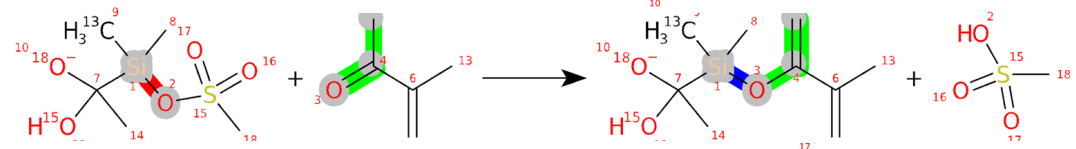

All Layers

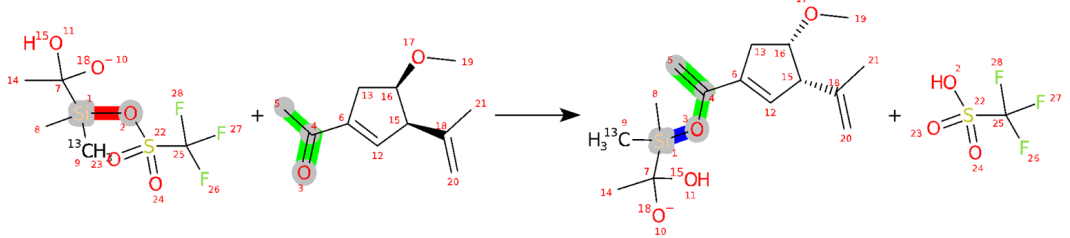

Figure 6: ReactionCode partial and complete decoding

The ReactionCode can be decoded by taking all or some layers. The layer 0 corresponds to the decoding of the reaction center only. Layers $0+1$ illustrates the decoding of the reaction center and all surrounding atoms in the remaining group having a depth equal to 1 , while layers $0+1+\mathrm{A}$ incorporates all surrounding atoms (remaining and leaving groups) with a depth of 1 . Layers $0+1+2$ and $0+1+2+\mathrm{A}+\mathrm{B}$ are considering all surrounding atoms present in the depth lower or equal to 2 . Layers $0+1+2+3+4+5$ is an example where the reaction is decoded in its entirety but without the leaving group. Finally, "All Layers" represents the decoding of the complete reaction. 
of the contents of a database. A clusterization of reaction data can also be useful in the context of machine learning, for trying to build the best possible training, test, and validation sets.

\subsection{Machine Learning}

The ReactionCode could be useful for machine learning applications as descriptors or directly for reaction prediction by predicting one or multiple layers. The ReactionCode describes the reaction center and its neighboring environment, which provides additional descriptors compared to current methods.

\subsection{Compression}

In the context of graph databases, the ReactionCode could be used as a tree structure where a node corresponds to a layer. This structure could improve the searching process but also help save disk usage because only the unique layers are stored. This structure permits one to retrieve and regenerate each reaction. Such a tree structure could be used to develop a reaction encoding process. Each layer could be transformed into a bit vector similarly to fingerprints used for molecules, which could allow one to compress a reaction and speed up the reaction comparison process.

\subsection{Evolution}

The ReactionCode was designed to be an upgradeable format. This format is open to the community, which can submit a new version. For instance, the aromatic bonds are encoded with the same character "9", which can fail to encode some tautomeric reactions. In a lot of mapped reactions, the correspondence of the Kekule versions in reactants and products is wrong, which will be considered as a change of the molecule and integrated into the reaction center. To avoid this problem, it is safest in most of the cases to adopt the aromatic annotation. However, if the user is sure of his/her mapping, this parameter can be easily changed and the bond will be encoded as single or double. Besides, it can be of interest to have the number of hydrogens in the ReactionCode for tautomeric reaction studies. This can be easily integrated into the reaction code by modifying one single parameter in the code.

\section{Conclusion}

ReactionCode has been implemented as a new, open source, versatile reaction format that avoids the drawbacks of others. The field of its possible applications is large and we believe that it can be profitable for the community working on reactions. Freely available and open source software has been developed to generate the ReactionCode from a reaction format, to convert the ReactionCode to 
a reaction format, and to use it as a reaction transform language. This program and the source code are available at https://cactus.nci.nih.gov/reactioncode. 


\section{References}

[1] G.D. Veith Anderson, E. and D. Weininger. SMILES: A line notation and computerized interpreter for chemical structures. Report No. EPA/600/M87/021. U.S. Environmental Protection Agency, Environmental Research Laboratory-Duluth, Duluth, MN 55804, Aug 1990.

[2] E. J. Corey, Richard D. Cramer, and W. Jeffrey Howe. Computer-assisted synthetic analysis for complex molecules. Methods and procedures for machine generation of synthetic intermediates. Journal of the American Chemical Society, 94(2):440-459, 1972.

[3] E. J. Corey and W. T. Wipke. Computer-assisted design of complex organic syntheses. Science, 166(3902):178-192, Oct 1969.

[4] E. J. Corey, W. Todd Wipke, Richard D. Cramer, and W. Jeffrey Howe. Computer-assisted synthetic analysis. Facile man-machine communication of chemical structure by interactive computer graphics. Journal of the American Chemical Society, 94(2):421-430, 1972.

[5] Arthur Dalby, James G. Nourse, W. Douglas Hounshell, Ann K. I. Gushurst, David L. Grier, Burton A. Leland, and John Laufer. Description of several chemical structure file formats used by computer programs developed at Molecular Design Limited. Journal of Chemical Information and Computer Sciences, 32(3):244-255, 1992.

[6] Aurélie de Luca, Dragos Horvath, Gilles Marcou, Vitaly Solov'ev, and Alexandre Varnek. Mining Chemical Reactions Using Neighborhood Behavior and Condensed Graphs of Reactions Approaches. Journal of Chemical Information and Modeling, 52(9):2325-2338, 2012. PMID: 22894688.

[7] Jean-Loup Faulon, Donald P. Visco, and Ramdas S. Pophale. The Signature Molecular Descriptor. 1. Using Extended Valence Sequences in QSAR and QSPR Studies. Journal of Chemical Information and Computer Sciences, 43(3):707-720, 2003. PMID: 12767129.

[8] Shinsaku Fujita. Description of organic reactions based on imaginary transition structures. 1. Introduction of new concepts. Journal of Chemical Information and Computer Sciences, 26(4):205-212, 1986.

[9] Marta Glavatskikh, Timur Madzhidov, Dragos Horvath, Ramil Nugmanov, Timur Gimadiev, Daria Malakhova, Gilles Marcou, and Alexandre Varnek. Predictive Models for Kinetic Parameters of Cycloaddition Reactions. Molecular Informatics, 38(1-2):1800077, 2019.

[10] G. Grethe, J. M. Goodman, and C. H. Allen. Internationa Chemical Identifier for Reactions (RInChI). J Cheminform, 5(1):45, Oct 2013. 
[11] S. Heller, A. McNaught, S. Stein, D. Tchekhovskoi, and I. Pletnev. InChI - the worldwide chemical structure identifier standard. J Cheminform, 5(1):7, Jan 2013.

[12] S. R. Heller, A. McNaught, I. Pletnev, S. Stein, and D. Tchekhovskoi. InChI, the IUPAC International Chemical Identifier. J Cheminform, 7:23, 2015.

[13] F. Hoonakker, N. Lachiche, A. Varnek, and A. Wagner. Condensed Graph of Reaction: Considering a Chemical Reaction As One Single Pseudo Molecule. Springer, Jul 2009.

[14] F.D. Culver Hunter, R.S. and A. Fitzgerald. SMILES User Manual. A Simplified Molecular Input Line Entry System. Includes extended SMILES for defining fragments. Review Draft, Internal Report, Montana State University, Institute for Biological and Chemical Process Control (IPA), Bozeman, MT, 1987.

[15] Philip N. Judson, Wolf-Dietrich Ihlenfeldt, Hitesh Patel, Victorien Delannée, Nadya Tarasova, and Marc C. Nicklaus. Adapting CHMTRN (CHeMistry TRaNslator) for a new use. 2020. DOI:10.26434/chemrxiv.11439984.v1.

[16] Hans Kraut, Josef Eiblmaier, Guenter Grethe, Peter Löw, Heinz Matuszczyk, and Heinz Saller. Algorithm for Reaction Classification. Journal of Chemical Information and Modeling, 53(11):2884-2895, 2013. PMID: 24102490 .

[17] Christophe Muller, Gilles Marcou, Dragos Horvath, João Aires-de Sousa, and Alexandre Varnek. Models for Identification of Erroneous Atom-toAtom Mapping of Reactions Performed by Automated Algorithms. Journal of Chemical Information and Modeling, 52(12):3116-3122, 2012. PMID: 23167287.

[18] David A. Pensak and E. J. Corey. LHASA - Logic and Heuristics Applied to Synthetic Analysis, volume 61, chapter 1, pages 1-32. ACS Symposium Series, 1977.

[19] Fiorella Ruggiu, Gilles Marcou, Alexandre Varnek, and Dragos Horvath. ISIDA Property-Labelled Fragment Descriptors. Molecular Informatics, 29(12):855-868, 2010.

[20] David Weininger. SMILES, a chemical language and information system. 1. Introduction to methodology and encoding rules. Journal of Chemical Information and Computer Sciences, 28(1):31-36, 1988.

[21] David Weininger, Arthur Weininger, and Joseph L. Weininger. SMILES. 2. Algorithm for generation of unique smiles notation. Journal of Chemical Information and Computer Sciences, 29(2):97-101, 1989. 
[22] E. L. Willighagen, J. W. Mayfield, J. Alvarsson, A. Berg, L. Carlsson, N. Jeliazkova, S. Kuhn, T. Pluskal, M. Rojas-Cherto, O. Spjuth, G. Torrance, C. T. Evelo, R. Guha, and C. Steinbeck. The Chemistry Development Kit (CDK) v2.0: atom typing, depiction, molecular formulas, and substructure searching. J Cheminform, 9(1):33, Jun 2017.

[23] W.Todd Wipke, Glenn I. Ouchi, and S. Krishnan. Simulation and evaluation of chemical synthesis - SECS: An application of artificial intelligence techniques. Artificial Intelligence, 11(1):173 - 193, 1978. Applications to the Sciences and Medicine.

[24] Mikiro Yanaka, Kazuhiko Nakamura, Azusa Kurumisawa, and W. Todd Wipke. Automatic knowledge base building for the organic synthesis design program (secs). Tetrahedron Computer Methodology, 3(6, Part A):359 375, 1990. 


\section{Supplementary information}
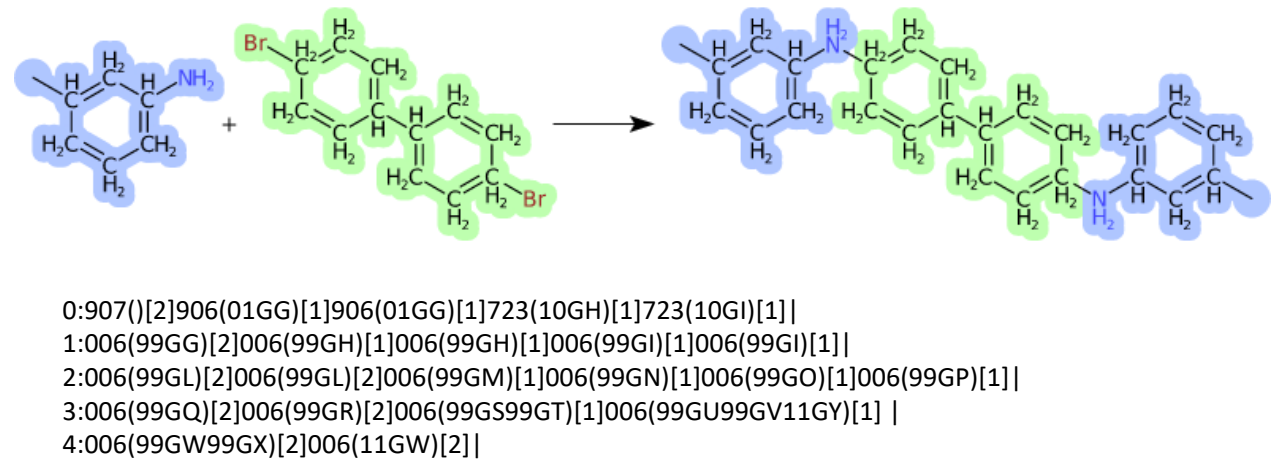

Figure S1: Conflict solver algorithm

The green molecule has $2 \mathrm{Br}$ atoms, which both react with the amine function of the blue molecule. The stoichiometry is 2 blue molecules for and 1 green molecule. Consequently, each atom of the green molecule has to be represented 2 times in the product, which is encoded by [2]. 


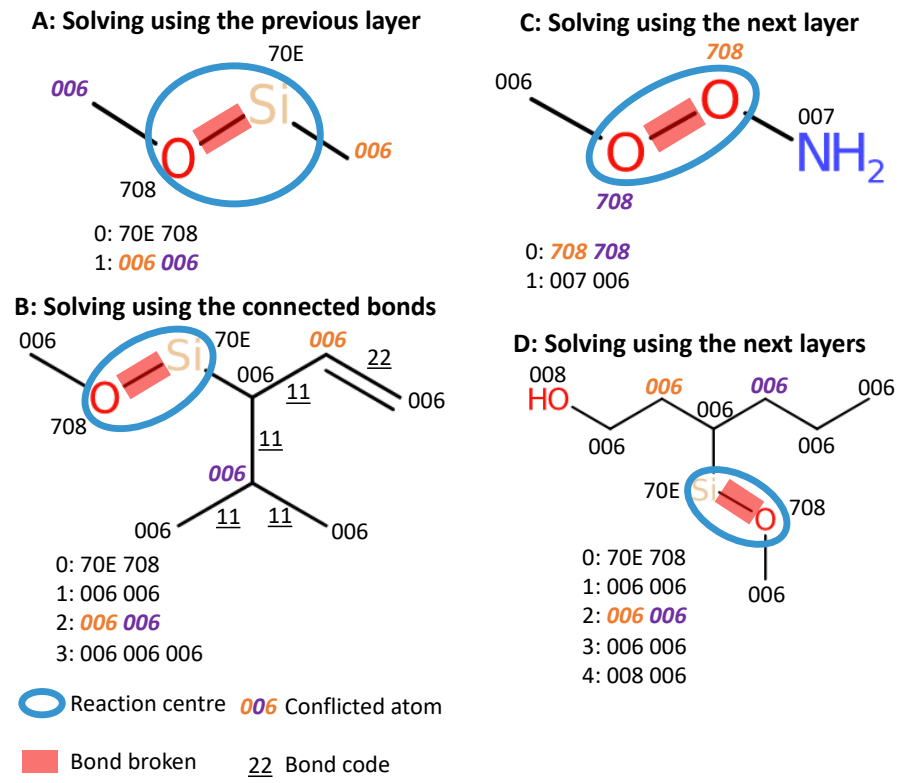

Figure S2: Conflict solver algorithm

After ranking each atom according to their codes, a conflict algorithm solves the conflicted atoms (i.e. those sharing the same code). This algorithm discriminates first by looking at atoms in the previous layers. If the conflict can not be solved, the bond type is used. If the two previous processes have failed, the priorities of the connected atoms in the next layer(s) are analyzed.

A: The carbon atom with an orange atom code has a higher priority because its connected atom ( $\mathrm{Si}$ atom with atom code $70 \mathrm{E}$ ) in the previous layer is ranked first, while the carbon atom in purple is connected to the atom at position 2 (O atom with atom code 708).

B: As the first method failed to solve the conflict, the bonds are taking into account. It takes into account the encoded bond types and the number of connected bonds with both layers. All bond codes related to the connected bond to the atom are reverse sorted and merged into a string. The merged code for carbon atom with an atom code in orange is 2211 and the one for carbon atom with an atom code in purple is 111111 . The carbon atom with an atom code in orange is ranked first because the priority of its merged code is higher $(2211>111111)$ by using the string comparison method: compareTo). C: As both previous methods failed to solve the conflict, the atom priorities are analyzed in the next layer. The oxygen atom with an atom code in orange has a higher priority because it is connected to a nitrogen atom having a higher priority than the neighbor (carbon atom) of the oxygen atom with an atom code in purple $(007>006)$.

D: In this example, the algorithm iterates over the next layers until being able to solve the conflict. The carbon atom with an atom code in orange has a higher priority because the oxygen atom in layer $n+2$ has a higher rank than the $\mathrm{n}+2$ carbon atom $(008>006)$. 


\begin{tabular}{|l|l|l|l|l|l|l|l|l|l|l|l|l|l|l|l|l|}
\hline & 0 & 1 & 2 & 3 & 4 & 5 & 6 & 7 & 8 & 9 & $\mathrm{a}$ & $\mathrm{b}$ & $\mathrm{c}$ & $\mathrm{d}$ & $\mathrm{e}$ & $\mathrm{f}$ \\
\hline 0 & 0 & 1 & 2 & 3 & 4 & 5 & 6 & 7 & 8 & 9 & 10 & 11 & 12 & 13 & 14 & 15 \\
\hline 1 & 16 & 17 & 18 & 19 & 20 & 21 & 22 & 23 & 24 & 25 & 26 & 27 & 28 & 29 & 30 & 31 \\
\hline 2 & 32 & 33 & 34 & 35 & 36 & 37 & 38 & 39 & 40 & 41 & 42 & 43 & 44 & 45 & 46 & 47 \\
\hline 3 & 48 & 49 & 50 & 51 & 52 & 53 & 54 & 55 & 56 & 57 & 58 & 59 & 60 & 61 & 62 & 63 \\
\hline 4 & 64 & 65 & 66 & 67 & 68 & 69 & 70 & 71 & 72 & 73 & 74 & 75 & 76 & 77 & 78 & 79 \\
\hline 5 & 80 & 81 & 82 & 83 & 84 & 85 & 86 & 87 & 88 & 89 & 90 & 91 & 92 & 93 & 94 & 95 \\
\hline 6 & 96 & 97 & 98 & 99 & 100 & 101 & 102 & 103 & 104 & 105 & 106 & 107 & 108 & 109 & 110 & 111 \\
\hline 7 & 112 & 113 & 114 & 115 & 116 & 117 & 118 & 119 & 120 & 121 & 122 & 123 & 124 & 125 & 126 & 127 \\
\hline 8 & 128 & 129 & 130 & 131 & 132 & 133 & 134 & 135 & 136 & 137 & 138 & 139 & 140 & 141 & 142 & 143 \\
\hline 9 & 144 & 145 & 146 & 147 & 148 & 149 & 150 & 151 & 152 & 153 & 154 & 155 & 156 & 157 & 158 & 159 \\
\hline a & 160 & 161 & 162 & 163 & 164 & 165 & 166 & 167 & 168 & 169 & 170 & 171 & 172 & 173 & 174 & 175 \\
\hline b & 176 & 177 & 178 & 179 & 180 & 181 & 182 & 183 & 184 & 185 & 186 & 187 & 188 & 189 & 190 & 191 \\
\hline c & 192 & 193 & 194 & 195 & 196 & 197 & 198 & 199 & 200 & 201 & 202 & 203 & 204 & 205 & 206 & 207 \\
\hline d & 208 & 209 & 210 & 211 & 212 & 213 & 214 & 215 & 216 & 217 & 218 & 219 & 220 & 221 & 222 & 223 \\
\hline e & 224 & 225 & 226 & 227 & 228 & 229 & 230 & 231 & 232 & 233 & 234 & 235 & 236 & 237 & 238 & 239 \\
\hline f & 240 & 241 & 242 & 243 & 244 & 245 & 246 & 247 & 248 & 249 & 250 & 251 & 252 & 253 & 254 & 255 \\
\hline
\end{tabular}

Table S1: Atom index encoding for reaction center and remaining group

\begin{tabular}{|c|c|c|c|c|c|c|c|c|c|c|c|c|c|c|c|c|c|c|c|c|}
\hline & $\mathrm{G}$ & $\mathrm{H}$ & 1 & $J$ & K & L & $\mathrm{M}$ & $\mathrm{N}$ & $\mathrm{O}$ & $\mathrm{P}$ & $\mathrm{Q}$ & $\mathrm{R}$ & $S$ & $\mathrm{~T}$ & $\mathrm{U}$ & V & W & $\mathrm{X}$ & $\mathrm{Y}$ & Z \\
\hline G & 1 & 8 & 3 & 4 & 5 & 6 & 7 & 8 & 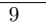 & 10 & 11 & 12 & 13 & 14 & 15 & 16 & 17 & 18 & 19 & 20 \\
\hline $\mathrm{H}$ & 21 & 22 & 23 & 24 & 25 & 26 & 27 & 28 & 29 & 30 & 31 & 32 & 33 & 34 & 35 & 36 & 37 & 38 & 39 & 40 \\
\hline I & 41 & 42 & 43 & 44 & 45 & 46 & 47 & 48 & 49 & 50 & 51 & 52 & 53 & 54 & 55 & 56 & 57 & 58 & 59 & 60 \\
\hline $\mathrm{J}$ & 61 & 62 & 63 & 64 & 65 & 66 & 67 & 68 & 69 & 70 & 71 & 72 & 73 & 74 & 75 & 76 & 77 & 78 & \begin{tabular}{|l|}
79 \\
\end{tabular} & 80 \\
\hline $\mathrm{K}$ & 81 & 82 & 83 & 84 & 85 & 86 & 87 & 88 & 89 & 90 & 91 & 92 & 93 & 94 & 95 & 96 & 97 & 98 & 99 & 100 \\
\hline $\mathrm{L}$ & 101 & 102 & 103 & 104 & 105 & 106 & 107 & 108 & 109 & 110 & 111 & 112 & 113 & 114 & 115 & 116 & 117 & 118 & \begin{tabular}{ll|}
119 \\
\end{tabular} & 120 \\
\hline $\mathrm{M}$ & 121 & 122 & 123 & 124 & 125 & 126 & 127 & 128 & 129 & 130 & 131 & 132 & 133 & 134 & 135 & 136 & 137 & 138 & \begin{tabular}{|l|l}
139 \\
\end{tabular} & 140 \\
\hline $\mathrm{N}$ & 141 & 142 & 143 & 144 & 145 & 146 & 147 & 148 & 149 & 150 & 151 & 152 & 153 & 154 & 155 & 156 & 157 & 158 & 159 & 160 \\
\hline $\mathrm{O}$ & 161 & 162 & 163 & 164 & 165 & 166 & 167 & 168 & 169 & 170 & 171 & 172 & 173 & 174 & 175 & 176 & 177 & 178 & \begin{tabular}{|l|}
179 \\
\end{tabular} & 180 \\
\hline $\mathrm{P}$ & 181 & 182 & 183 & 184 & 185 & 186 & 187 & 188 & 189 & 190 & 191 & 192 & 193 & 194 & 195 & 196 & 197 & 198 & \begin{tabular}{|l|l}
199 \\
\end{tabular} & 200 \\
\hline Q & 201 & 202 & 203 & 204 & 205 & 206 & 207 & 208 & 209 & 210 & 211 & 212 & 213 & 214 & 215 & 216 & 217 & 218 & 219 & 220 \\
\hline $\mathrm{R}$ & 221 & 222 & 223 & 224 & 225 & 226 & 227 & 228 & 229 & 230 & 231 & 232 & 233 & 234 & 235 & 236 & 237 & 238 & \begin{tabular}{|l|l}
239 \\
\end{tabular} & 240 \\
\hline $\mathrm{S}$ & 241 & 242 & 243 & 244 & 245 & 246 & 247 & 248 & 249 & 250 & 251 & 252 & 253 & 254 & 255 & 256 & 257 & 258 & 259 & 260 \\
\hline $\mathrm{T}$ & 261 & 262 & 263 & 264 & 265 & 266 & 267 & 268 & 269 & 270 & 271 & 272 & 273 & 274 & 275 & 276 & 277 & 278 & \begin{tabular}{|l|}
279 \\
\end{tabular} & 280 \\
\hline $\mathrm{U}$ & 281 & 282 & 283 & 284 & 285 & 286 & 287 & 288 & 289 & 290 & 291 & 292 & 293 & 294 & 295 & 296 & 297 & 298 & \begin{tabular}{|l|l}
299 \\
\end{tabular} & 300 \\
\hline V & 301 & 302 & 303 & 304 & 305 & 306 & 307 & 308 & 309 & 310 & 311 & 312 & 313 & 314 & 315 & 316 & 317 & 318 & 319 & 320 \\
\hline W & 321 & 322 & 323 & 324 & 325 & 326 & 327 & 328 & 329 & 330 & 331 & 332 & 333 & 334 & 335 & 336 & 337 & 338 & 339 & 340 \\
\hline $\mathrm{X}$ & 341 & 342 & 343 & 344 & 345 & 346 & 347 & 348 & 349 & 350 & 351 & 352 & 353 & 354 & 355 & 356 & 357 & 358 & 359 & 360 \\
\hline $\mathrm{Y}$ & 361 & 362 & 363 & 364 & 365 & 366 & 367 & 368 & 369 & 370 & 371 & 372 & 373 & 374 & 375 & 376 & 377 & 378 & 379 & 380 \\
\hline Z & 381 & 382 & 383 & 384 & 385 & 386 & 387 & 388 & 389 & 390 & 391 & 392 & 393 & 394 & 395 & 396 & 397 & 398 & \begin{tabular}{|l|l}
399 \\
\end{tabular} & 400 \\
\hline
\end{tabular}

Table S2: Atom index encoding for leaving group 


\begin{tabular}{|c|c|c|c|c|}
\hline Symbol & Type & Shorthand & Numeric shorthand & encoding \\
\hline @TH1 & Tetrahedral & ANTI CLOCKWISE (=LEFT) & 1 & 1 \\
\hline @TH2 & Tetrahedral & CLOCKWISE (=RIGHT) & 2 & 2 \\
\hline @AL1 & ExtendedTetrahedral & ANTI CLOCKWISE (=LEFT) & 1 & 3 \\
\hline @AL2 & ExtendedTetrahedral & CLOCKWISE (=RIGHT) & 2 & 4 \\
\hline @DB1 & DoubleBond & OPPOSITE & 1 & 5 \\
\hline @DB2 & DoubleBond & TOGETHER & 2 & 6 \\
\hline$@$ CT1 & ExtendedCisTrans & OPPOSITE & 1 & 7 \\
\hline @CT2 & ExtendedCisTrans & TOGETHER & 2 & 8 \\
\hline @SP1 & SquarePlanar & & 1 & 9 \\
\hline @SP2 & SquarePlanar & & 2 & $\mathrm{~A}$ \\
\hline @SP3 & SquarePlanar & & 3 & $\mathrm{~B}$ \\
\hline @TB1 & TrigonalBipyramidal & ANTI CLOCKWISE (=LEFT) & 1 & $\mathrm{C}$ \\
\hline @TB2 & TrigonalBipyramidal & CLOCKWISE (=RIGHT) & 2 & $\mathrm{D}$ \\
\hline$@ \mathrm{OH} 1$ & Octahedral & ANTI CLOCKWISE (=LEFT) & 1 & $\mathrm{E}$ \\
\hline$@ \mathrm{OH} 2$ & Octahedral & CLOCKWISE (=RIGHT) & 2 & $\mathrm{~F}$ \\
\hline @AP1 & Atropisomeric & ANTI CLOCKWISE (=LEFT) & 1 & G \\
\hline @AP2 & Atropisomeric & CLOCKWISE (=RIGHT) & 2 & $\mathrm{H}$ \\
\hline
\end{tabular}

Table S3: Atom stereochemistry encoding

\begin{tabular}{|l|l|}
\hline Type & encoding \\
\hline E & 1 \\
\hline Z & 2 \\
\hline DOWN INVERTED & 3 \\
\hline DOWN & 4 \\
\hline UP INVERTED & 5 \\
\hline UP & 6 \\
\hline E or Z & 7 \\
\hline UP or DOWN & 8 \\
\hline UP or DOWN INVERTED & 9 \\
\hline
\end{tabular}

Table S4: Bond stereochemistry encoding 


\begin{tabular}{|l|l|l|l|l|}
\hline C or i & encoding & & C or i & encoding \\
\hline 1 & I & -1 & H \\
\hline 2 & J & -2 & G \\
\hline 3 & K & -3 & F \\
\hline 4 & L & -4 & E \\
\hline 5 & M & -5 & D \\
\hline 6 & N & -6 & C \\
\hline 7 & O & -7 & B \\
\hline 8 & $\mathrm{P}$ & -8 & $\mathrm{~A}$ \\
\hline 9 & $\mathrm{Q}$ & -9 & 9 \\
\hline 10 & $\mathrm{R}$ & -10 & 8 \\
\hline 11 & $\mathrm{~S}$ & -11 & 7 \\
\hline 12 & $\mathrm{~T}$ & -12 & 6 \\
\hline 13 & $\mathrm{U}$ & -13 & 5 \\
\hline 14 & $\mathrm{~V}$ & -14 & 4 \\
\hline 15 & $\mathrm{~W}$ & -15 & 3 \\
\hline 16 & $\mathrm{X}$ & -16 & 2 \\
\hline 17 & $\mathrm{Y}$ & -17 & 1 \\
\hline
\end{tabular}

Table S5: Charge and Isotope encoding

\begin{tabular}{|c|l|}
\hline 1 & SINGLE \\
\hline 2 & DOUBLE \\
\hline 3 & TRIPLE \\
\hline 4 & QUADRUPLE \\
\hline 5 & QUINTUPLE \\
\hline 6 & SEXTUPLE \\
\hline 9 & AROMATIC \\
\hline 0 & NONE \\
\hline
\end{tabular}

Table S6: Bond order encoding 


\begin{tabular}{|l|l|l|}
\hline score & meaning & encoding \\
\hline 0 & unmarked & 0 \\
\hline-1 & not a reaction centre & 0 \\
\hline 1 & a reaction centre & 1 \\
\hline 4 & bond order changes & 4 \\
\hline 6 & bond broken & 6 \\
\hline 8 & bond made & 8 \\
\hline 5 & $4+1$ (is a center and order changes) & 5 \\
\hline 7 & $6+1$ (is a center and bond broken) & 7 \\
\hline 9 & $8+1$ (is a center and bond made) & 9 \\
\hline 10 & $6+4$ (bond is both broken and its order changes) & A \\
\hline 11 & $10+1$ (is a center, bond broken and order changes) & B \\
\hline 12 & $8+4$ (bond is both made and its order changes) & C \\
\hline 13 & $12+1$ (is a center, bond made and order changes) & D \\
\hline
\end{tabular}

Table S7: Bond change status encoding

-1 will be increased to 0

10 will be reduced to 4

11 will be reduced to 5

12 will be reduced to 8

13 will be reduced to 9 


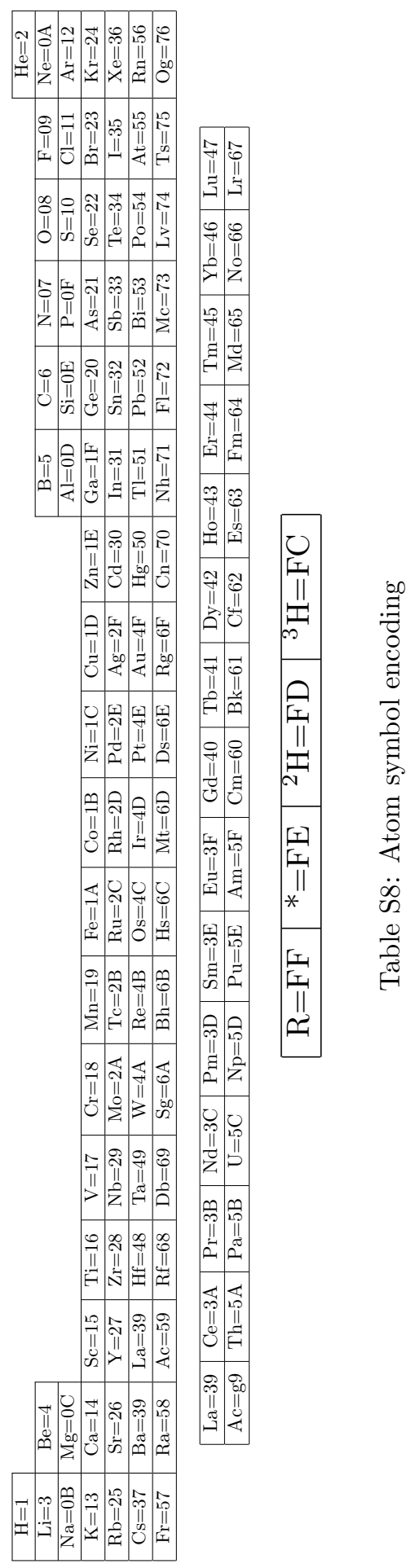

